\title{
ESTIMATION DE LA QUALITE DES EAUX DE LA SOMME PAR L'ETUDE DES PEUPLEMENTS ALGAUX BENTHIQUES
}

\author{
par J.-P. Descy $\left(^{*}\right)$ \\ Département de Botanique \\ Université de Liège, \\ Sart Tilman \\ B-4 000 Liège, Belgique
}

\section{RESUME}

Une étude de la végétation algale benthique de la Somme, effectuée en septembre 1974, a permis d'établir une estimation de la qualité biologique des eaux de la Somme. La méthode utilisée, mise au point au cours de recherches dans la Meuse et la Sambre belges, est basée sur l'étude quantitative des peuplements de Diatomées benthiques.

\section{1. - INTRODUCTION}

Dans le but de tester la possibilité de généraliser une méthodologie d'estimation biologique de la qualité des eaux mise au point au cours de travaux sur la Meuse et la Sambre belges (DESCY, 1975 et sous presse), l'étude d'une autre rivière relativement proche par ses caractéristiques physico-chimiques de base présentait un grand intérêt. Suite à des contacts avec l'Université de Picardie à Amiens, l'occasion nous a été donnée d'étudier la végétation algale benthique de la Somme. Le choix de la Somme présentait, du point de vue pratique, l'avantage considérable que cette rivière, ainsi que l'ensemble du bassin hydragraphique géré par l'Agence de Bassin Artois-Picardie, sont l'objet de nombreuses campagnes d'analyses physico-chimiques des eaux. De cette façon. la qualité physico-chimique des eaux de la Somme au cours de ces dernières années est bien connue - au moins au niveau des principaux facteurs polluants et nous avons voulu profiter de ce cadre pour entreprendre des analyses bio-

(*) Assistant au Centre de Recherches Fondamentales sur les Bioindicateurs de la Pollution des Milieux Continentaux (FRFC). 
logiques du même type que celles réalisées dans la Meuse et la Sambre. Cette ètude présente donc à notre point de vue un double intérêt: outre celui, sur un plan général, de décrire la végétation algale benthique de la Somme, elle nous permettait, sans devoir réaliser nous-mêmes des analyses physico-chimiques longues et détaillées, de vérifier, dans une rivière appartenant à un autre bassin hydrographique, l'applicabilité de la méthodologie d'estimation de la qualité des eaux mise au point dans la Meuse et la Sambre belges.

D'après le document *Etat de pollution des cours d'eau. (Agence de Bassin Artois-Picardie, 1974) et d'après quelques analyses complémentaires effectuées en septembre 1974, la situation physico-chimique de la Somme peut se résumer de la façon suivante : en aval de la première station (Morcourt), considérée comme "normale», survient après la traversée de Saint-Quentin une pollution organique et chimique très importante, qui diminue en aval mais n'est complètement résorbée qu'au début du canal de la Somme, à Cappy $\left(\mathrm{SO}_{5}\right)$ (Fig. 1). Une bonne qualité des eaux se maintient ensuite jusqu'à Amiens, puis une nouvelle dégradation de moindre importance et de nature différente apparait jusqu'à Abbeville : elle se manifeste par un léger accroissement de la pollution organique et, pour autant que la traduction des résultats en flux moyens puisse permettre d'en juger, par une augmentation des matières en suspension et des chlorures.

Quelques paramètres importants nous manquent cependant pour confirmer ou compléter cette description : la température, le $\mathrm{pH}$, la conductivité, les phosphates, l'alcalinité, les différentes formes d'azote...

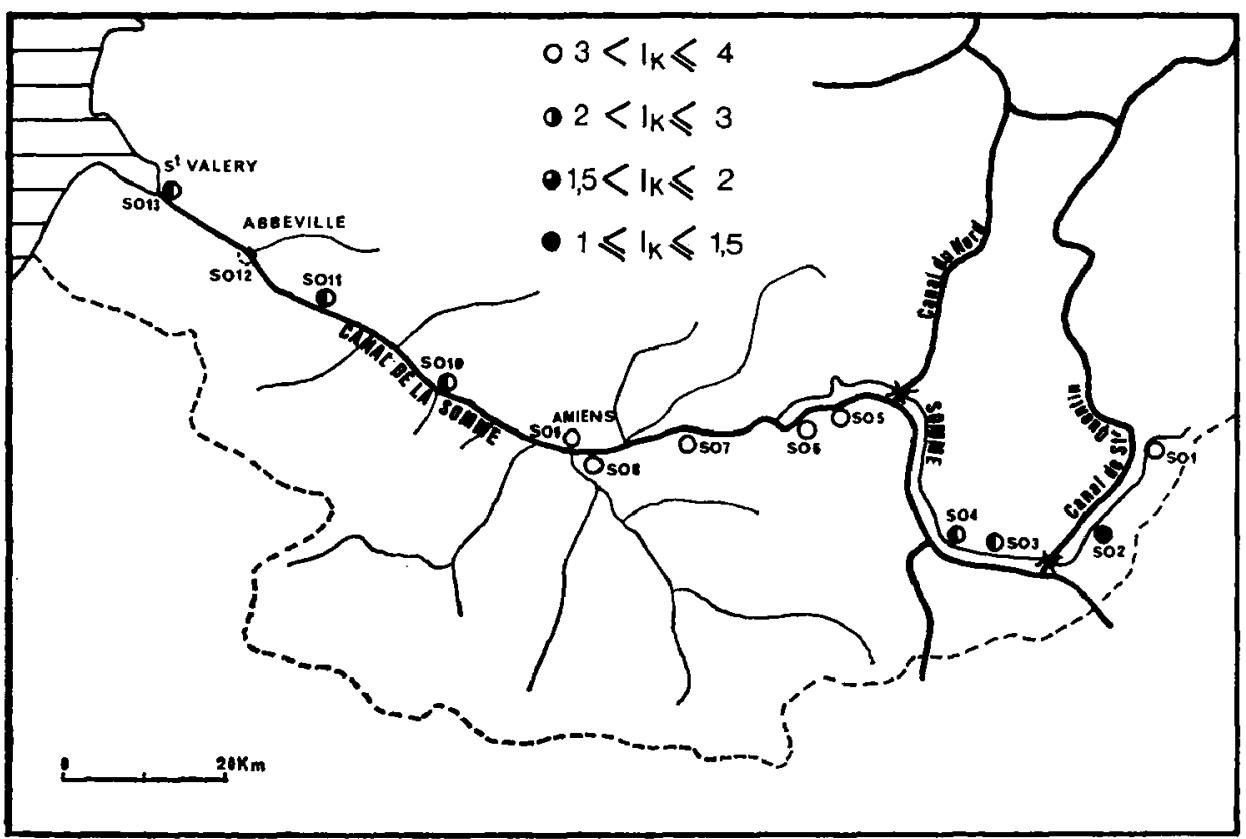

Figure 1 : Carte simplifiée du cours de la Somme, situant les stations d'échantillonnage en septembre 1974. Les symboles permettent de visualiser la qualité des eaux aux différentes stations, d'après les valeurs de l'indice biotique basè sur les Diatomées. 


\section{2. - etude de la vegetation algale benthiQue}

Cette étude, réalisée en septembre 1974 dans 13 stations de prélèvements (voir carte fig. 1) réparties sur tout le cours de la Somme, a permis de décrire et de caractériser la végétation algale macroscopique de la Somme (algues filamenteuses et algues bleues). Les peuplements de Diatomées benthiques ont fait l'objet d'études plus approfondies, débouchant notamment sur des estimations du développement des principales espèces dans chaque station. Sur l'ensemble des récoltes, plus de 205 taxons (espèces et variétés) sont identifiés : la majorité sont des espèces basophiles, caractéristiques des eaux calcaires et eutrophes (association à Diatoma vulgare et Melosira varians 1954).

Les estimations de densité d'une cinquantaine de Diatomées ont permis de suivre leurs distributions sur le cours de la Somme, qui apparaissent très différenciées par suite de l'existence des pollutions. Par une étude statistique plus poussée de ces distributions au moyen d'une technique d'analyse multivariable (analyse factorielle des correspondances), différents groupements de Diatomées sont mis en évidence, ainsi que les facteurs responsables de leur formation. Dans le cas de la Somme, le principal facteur de modification des peuplements est la pollution sous ses différents aspects; l'augmentation de salinité à l'embouchure de la Somme (Saint-Valéry) intervient également.

Une seconde technique d'analyse multivariable, l'analyse en composantes principales, appliquée aux données quantitatives (voir DESCY, 1975 et sous presse) permet d'obtenir une classification des Diatomées en fonction de leur sensibilité à la pollution des eaux : la * polluosensibilité - de chaque espèce peut ainsi être estimée en lui attribuant un indice correspondant à son degré de résistance à la pollution. Cette analyse fournit également une * ordination * des stations échantillonnées suivant le degré de pollution qui y est atteint.

\section{3. - APPLICATION a la somme de la methodologie D'ESTIMATION DE LA QUALITE DES EAUX BASEE SUR LES DIATOMEES BENTHIQUES}

Cette estimation consiste à calculer un "indice biotique " pour chaque station, en utilisant le développement relatif et la polluosensibilité des Diatomées benthiques suivant la formule:

$$
\begin{aligned}
& \text { p } \\
& \Sigma D_{k k}, i_{1} \\
& j=1 \\
& l_{k}=\frac{J=1}{p} \\
& \Sigma D_{1 x} \\
& j=1
\end{aligned}
$$

oú $\mathrm{K} .=$ indice biotique de la station $\mathrm{k}$

$D_{1 k}=$ densité (absolue ou relative) de l'espèce $j$ dans la station $k$

$i,=$ indice de polluosensibilité de l'espèce $\mathbf{j}$. 
Les valeurs de l'indice sont comprises entre 1 et 4 et correspondent à divers niveaux de qualité et de pollution des eaux (voir DESCY, 1975 et sous presse).

Pour effectuer une comparaison directe, le calcul de l'indice des populations diatomiques a été effectué d'une part d'après les indices de polluosensibilité résultant directement de l'analyse des Diatomées du périphyton de la Somme, ainsi que d'autre part sur la base des indices similaires établis pour les espèces communes avec la Meuse et la Sambre belges; pour les quelques espèces représentées uniquement dans la Somme, ce sont bien entendu les indices de polluosensibilité déterminés par la présente étude qui ont été utilisés dans les deux cas. Les indices $I_{k}$ obtenus de cette façon sont présentés au tableau 1 et la fig. 2 réunit les deux courbes d'évolution de l'indice biotique.

Les deux courbes montrent une évolution remarquablement semblable : Morcourt $\left(\mathrm{SO}_{1}\right)$ et les stations comprises entre Frise $\left(\mathrm{SO}_{5}\right)$ et Amiens ( $\left.\mathrm{SO}_{9}\right)$ présentent des valeurs de $l_{k}$ proches de 4 et donc des communautés témoignant d'une bonne qualité des eaux. La pollution importante survenant à Séraucourt $\left(\mathrm{SO}_{2}\right)$ se traduit par une chute de l'indice à une valeur proche de 1 (correspondant à l' « association * à dominance de Nitzschia palea). Une épuration efficace apparait ensuite jusque $\mathrm{Ham}\left(\mathrm{SO}_{3}\right)$, suivie d'une nouvelle dégradation à Offoy $\left(\mathrm{SO}_{4}\right)$. La pollution, complètement résorbée dans le canal de la Somme, réapparait en aval $d$ 'Amiens avec une importance moindre, ainsi que l'indiquent les valeurs de $l_{k}$ à Picquigny $\left(\mathrm{SO}_{10}\right)$ et Pont-Rémy $\left(\mathrm{SO}_{11}\right)$. Des valeurs plus basses s'observent à Saint-Valèry, qui sont sans doute plus une conséquence de l'augmentation de salinité que de la pollution.

La carte de la fig. 1 permet de visualiser, par un procédé simplifié, la qualité biologique des eaux dans chaque station.

\section{TABLEAU 1}

Indices $I_{k}$, d'après les indices de polluosensibilité des Diatomées établis dans la Somme (col. 1) et dans la Sambre et la Meuse (col. 2)

\begin{tabular}{lll}
\hline $\mathrm{SO}_{1}$ & 3.96 & 3.96 \\
$\mathrm{SO}_{2}$ & 1.04 & 1.04 \\
$\mathrm{SO}_{3}$ & 2.75 & 2.9 \\
$\mathrm{SO}_{4}$ & 2.03 & 2.04 \\
$\mathrm{SO}_{5}$ & 3.91 & 3.52 \\
$\mathrm{SO}_{6}$ & 3.88 & 3.74 \\
$\mathrm{SO}_{7}$ & 3.94 & 3.92 \\
$\mathrm{SO}_{8}$ & 3.85 & 3.83 \\
$\mathrm{SO}_{9}$ & 3.85 & 3.86 \\
$\mathrm{SO}_{10}$ & 2.58 & 2.57 \\
$\mathrm{SO}_{11}$ & 2.85 & 2.73 \\
$\mathrm{SO}_{12}$ & - & - \\
$\mathrm{SO}_{13}$ & 2.09 & 1.42 \\
\hline
\end{tabular}




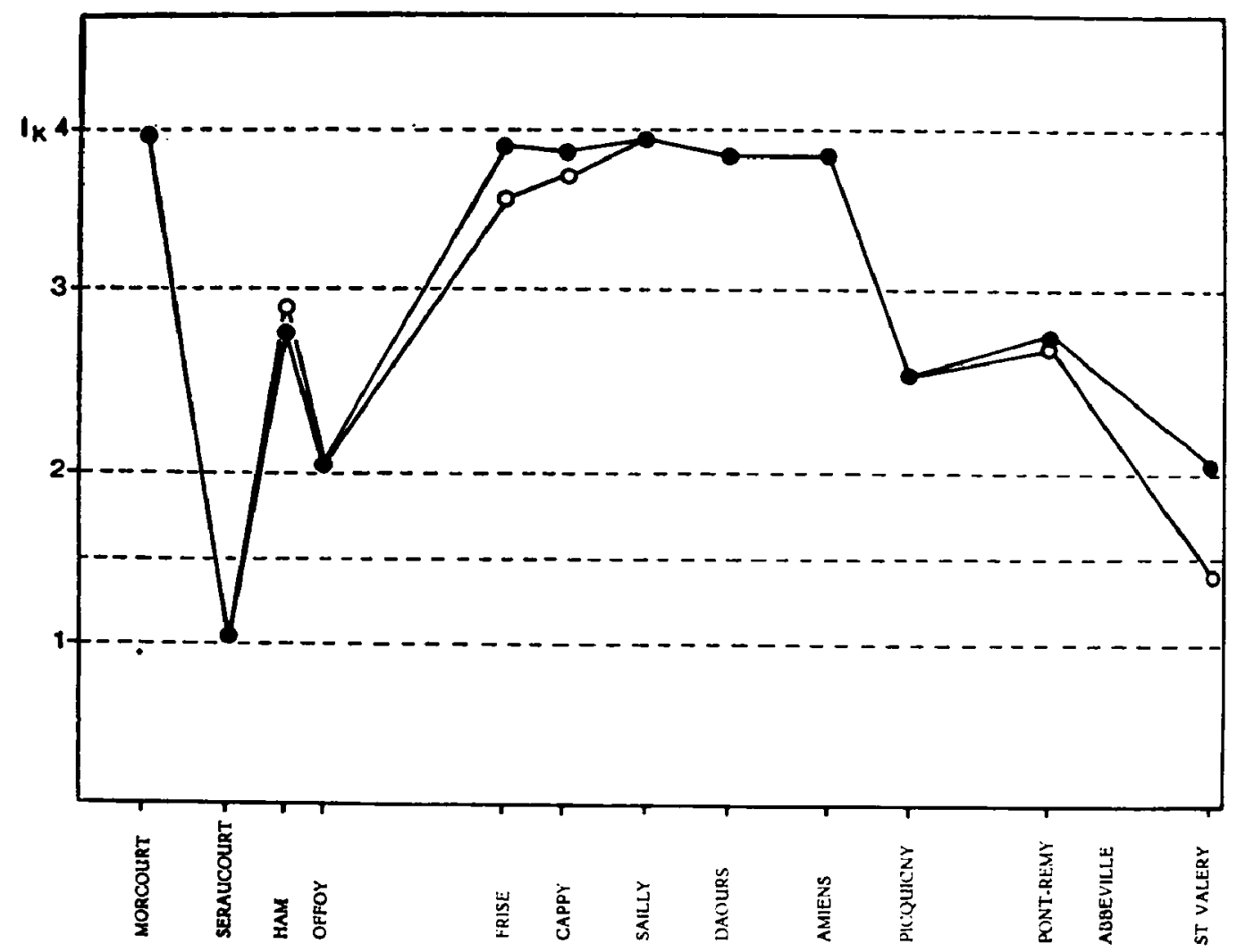

Figure 2 : Evolution de l'indice $I_{k}$ dans la Somme, calculé d'après les indices de polluosensibilité des Diatomées résultant de l'analyse de la Somme (points noirs), et d'après les indices similaires établis pour les espèces communes avec la Meuse et la Sambre (points blancs). Les traits discontinus délimitent les différents niveaux de qualité des eaux reconnaissables d'après l'indice biotique.

\section{TRAVAUX CITES}

DESCY J.-P., 1975. Etude quantitative du peuplement algal benthique en vue de l'établissement d'une méthodologie d'estimation biologique de la qualité des eaux courantes. Thèse Doct. Sc. Bot., Liège, 173 pp.

DESCY J.-P. (sous presse). Utilisation des algues benthiques comme indicateurs biologiques de la qualité des eaux courantes. In * Pollution des eaux continentales $*$, edit. P. Pesson, Paris. 